\title{
Detection of Low-Contrast Objects with a Focusing Transmitarray
}

\author{
Germán León, Enrique G. Plaza, Susana Loredo, Marcos R. Pino \\ Area of Signal Theory and Communications, Universidad de Oviedo, Gijón, Spain \\ \{gleon, egplaza, sloredo,mpino\}@tsc.uniovi.es
}

\begin{abstract}
A Focusing Transmitarray is a quasi-periodic planar lens that is able to focus the energy of a feed at a spot in the Near Field of the lens. In this work, this kind of lens has been used for detection under low contrast conditions. The use of this lens allows non-contact measurements, therefore increasing the sensitivity of a detection system. The resolution of the lens has been experimentally studied by measuring the separation between two metal strips (high contrast) and between two plastic strips on a foam layer (low contrast). This measurement set-up has been used to detect small objects on a metal plate. This screen reflects all the incident radiation hiding the presence of any small object. Using a focusing lens and a proper, but simple, calibration it is possible to find the object without any ambiguity.
\end{abstract}

Index Terms-Transmitarray, focusing energy, non-contact detection.

\section{INTRODUCTION}

$\mathrm{T}$ RANSMITARRAY antennas (TA) can be described as planar lenses that transform the spherical wave front of a feed into a different one. They are based on a quasi-periodical lattice formed by more than two stacked dielectric layers, where a unitcell is repeated in two dimensions of the space. TAs have several advantages: light weight, simplicity to be manufactured and blockage free, mainly. They are good candidates as high gain antennas $[1,2]$. However, they can also be used to focus the electromagnetic energy at a spot near the antenna.

Focusing antennas have been used to increase the sensitivity of RFID systems [3], radiometers [4], for industrial inspection $[5,6]$, etc. These antennas are microstrip patch arrays fed by a complex network, whose losses increase as frequency does. To overcome this problem, Fresnel zone plates have been used for focusing [7]. Theses lenses are composed of a set of alternative transparent - opaque annuli. In this case, half of the power emitted by the feed is radiated backwards, decreasing drastically the efficiency of the system. Nevertheless, TA lens are quasi-transparent, that is, almost all the power is re-radiated to the working zone.
In this contribution, a focusing TA (FTA) antenna is proposed as a system to detect low-permittivity objects. In this work, X-band has been chosen as a trade-off solution penetrating depth and resolution characteristics. The TA based system allows real time measurement without the use of any signal processing.

\section{Resolution Study OF THE Focusing TA}

A two layer FTA has been designed to focus the energy at a distance of $135 \mathrm{~mm}$ from the lens, working at $10 \mathrm{GHz}$. It was also manufactured and measured. The characteristics of the cell and the designing process can be found in [8-9]. The TA lens increases the field in the focusing point by $14 \mathrm{~dB}$ when compared to the field generated by the feed alone. Furthermore, the system has a $1 \mathrm{~dB}$ spot of $16 \mathrm{~mm}$ and a $3 \mathrm{~dB}$ spot of $26 \mathrm{~mm}$, approximately equal in both axes.

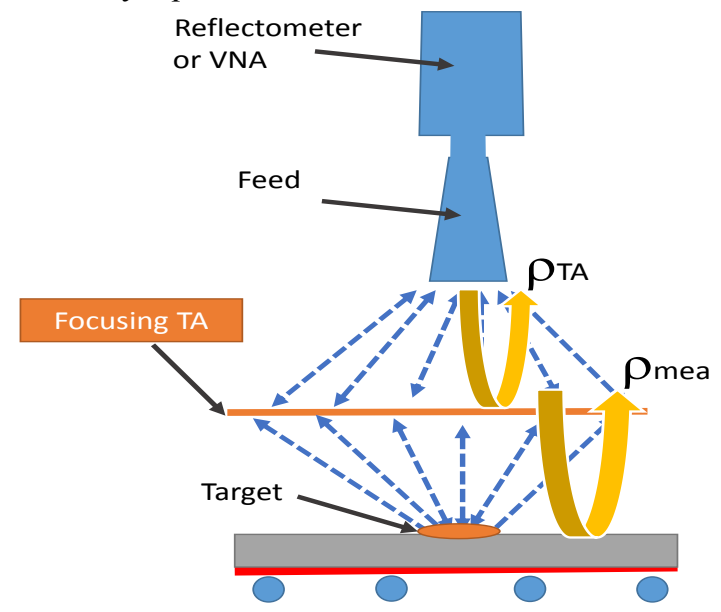

Fig. 1. General scheme of a detection system based on a focusing TA lens.

In Fig. 1, a scheme of a generic resolution set-up is shown. The FTA system is connected to a vector network analyzer to measure the reflection coefficient of the stand-alone device $\left(\rho_{T A}\right)$, due to the mismatching of the feed and the non-total transparence of the lens. In the measurement campaign, a sample under test (SUT) is illuminated by the FTA and the same 
FTA collects the backward radiation, so a reflection coefficient $\rho_{\text {mea }}$ is measured. This coefficient is the sum of the backward radiation of the SUT and the backward radiation from the FTA. Thus, the reflection coefficient of the SUT, can be calculated as

$$
\rho_{S U T}=\rho_{\text {mea }}-\rho_{T A}
$$

In the first experiment (Fig. 2), two metal strips, of 18mils height, are placed on a foam layer. Two different distances between the two strips (d) were measured: $15 \mathrm{~mm}$ and $10 \mathrm{~mm}$. The results of the $\left|\rho_{\text {SUT }}\right|$ are shown in Fig. 3. The resolution can be defined as the capacity to distinguish two targets close to each other. In this case, the threshold is $-2 \mathrm{~dB}$, that is, the end of a target is considered when $\left|\rho_{\text {SUT }}\right|$ is $2 \mathrm{~dB}$ below the maximum. Then, when $\mathrm{d}=15 \mathrm{~mm}$ two objects can be detected. However, if the separation between strips decreases $(\mathrm{d}=10 \mathrm{~mm})$, a local minimum $2 \mathrm{~dB}$ below the maximum cannot be found. In that case, resolution is not high enough, and only one target can be found. It must be noted that this resolution $(\mathrm{d}=15 \mathrm{~mm})$ matches with the 1-dB spot width of the FTA.

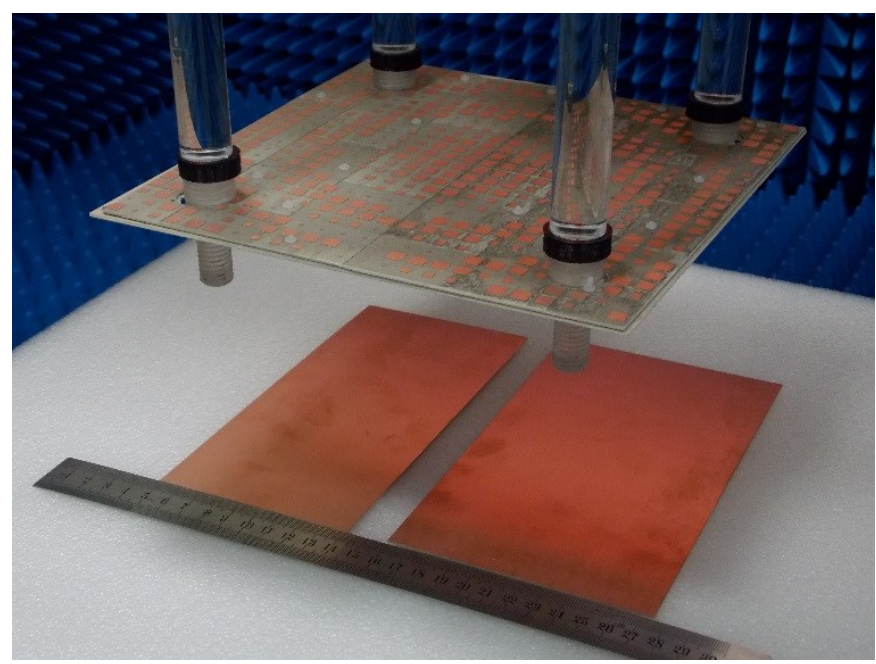

Fig. 2. Measurement set-up to FTA resolution characterization

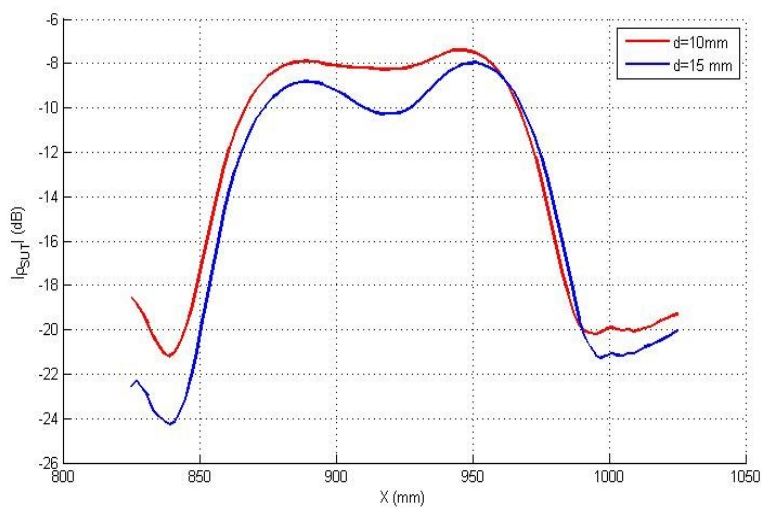

Fig. 3. Measured SUT reflection coefficient of metal strips with different separation distance.

The same experiment has been done replacing the metal strips by two plastic ones. These strips are manufactured with polylactide (PLA) being $40 \mathrm{~mm}$ length and $2 \mathrm{~mm}$ height. PLA is a dielectric material with a relative permittivity of 2.5 and a loss tangent of 0.005 . The strips were placed on a foam surface and the separation distances were the same as the previous case. The contrast between the air and the PLA is considerably low, nonetheless it can be seen in Fig. 4 that it is possible to find the edges of the plastic strips when the distance is $15 \mathrm{~mm}$. The resolution is lost below this separation distance, as in the metal strip case.

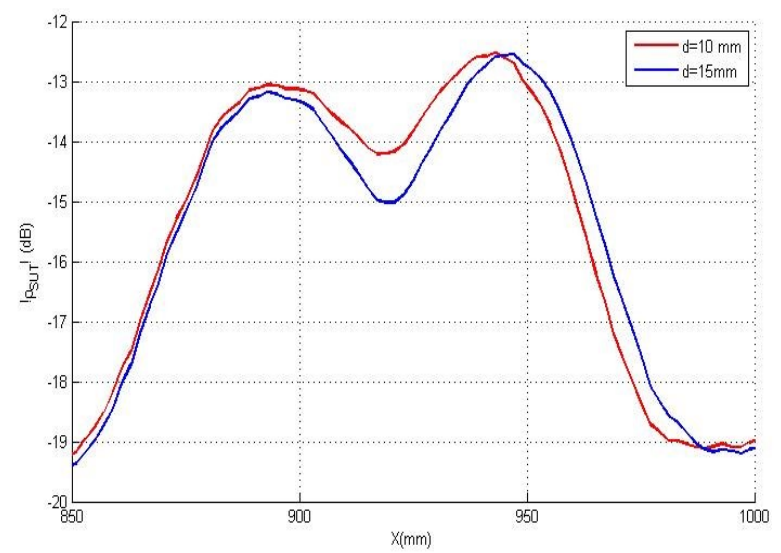

Fig. 4. Measured SUT reflection coefficient of plastic strips with different separation distance.

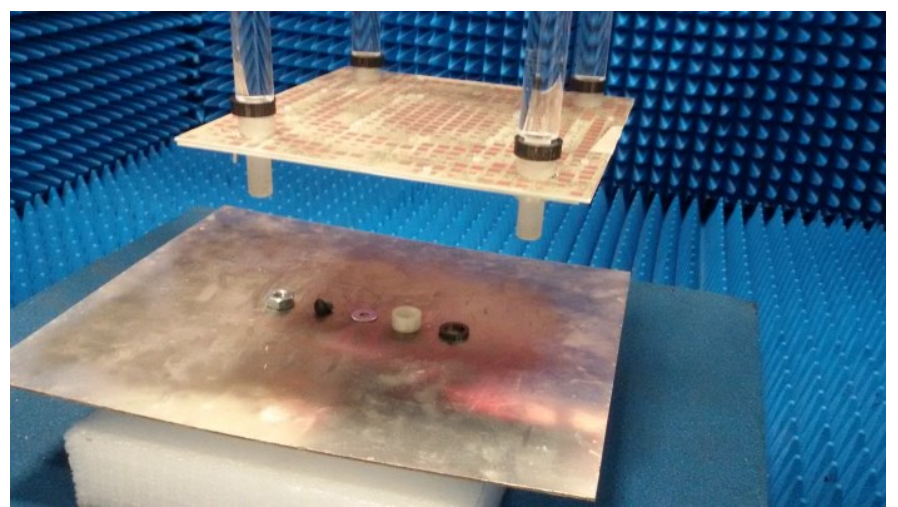

Fig. 5. Photography of different screws, nuts and washer used to evaluate the proposed detection system.

\section{DETECTION OF SMALL TARGETS ON A METAL PLATE}

Different experiments have been carried out to show the potential use of the FTA system as a detection device. Fig. 5 shows some of the targets studied. These targets have been measured on a metal plate. This metal plate reflects all the impinging power and hides any anomalies that could appear. That is, there is low contrast between the metal plate and the anomalies or the target. A FTA increases the power that impact on the SUT and, also, increases the received power from the target. In this experiment, the reflection coefficient from the metal plate alone $\left(\rho_{\text {met }}\right)$ was calibrated to eliminate its radiation in order to detect the targets, using an expression similar to (1):

$$
\rho_{\text {SUT }}=\rho_{\text {mea }}-\rho_{\text {met }}
$$

In the first experiment, a metal screw was placed on the plate. 
The measured reflection coefficient has been plot in a dotted line in Fig. 6. This coefficient changes less than $1 \mathrm{~dB}$ with the presence of the screw. However, removing the contribution of the metal radiation, it can be seen a variation of $10 \mathrm{~dB}$ in the $\left|\rho_{S U T}\right|$ coefficient, so the screw is clearly detected.

In a second experiment, instead of a screw, a metal washer of $1 \mathrm{~mm}$ height and $1 \mathrm{~cm}$ of diameter has been used. The variations of $\left|\rho_{\text {met }}\right|$ are below $0.5 \mathrm{~dB}$ and are very similar to variations produced by other effects (noise, vibrations of the set-up...). However, $\left|\rho_{S U T}\right|$ shows an increased variation of more than $5 \mathrm{~dB}$ when the washer is measured.

Finally, a dielectric nut, made with PLA, was placed on the metal plate. As in the previous cases, the variations in $\left|\rho_{\text {met }}\right|$ cannot show the presence of any object. Nevertheless, the parameter $\left|\rho_{S U T}\right|$ shows clearly the presence of an object, with variations of $15 \mathrm{~dB}$.

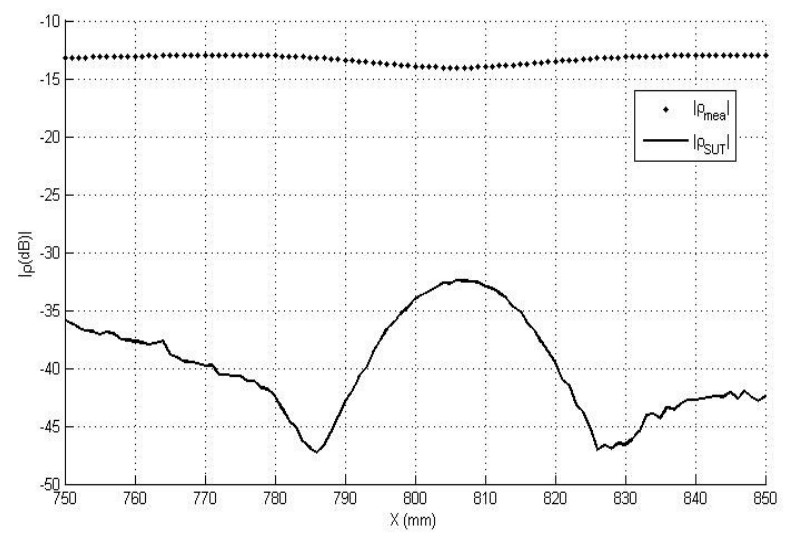

Fig. 6. Measurement of the SUT reflection coefficient of a metal screw on a metal plate.



Fig. 7. Measurement of the SUT reflection coefficient of a metal washer on a metal plate.

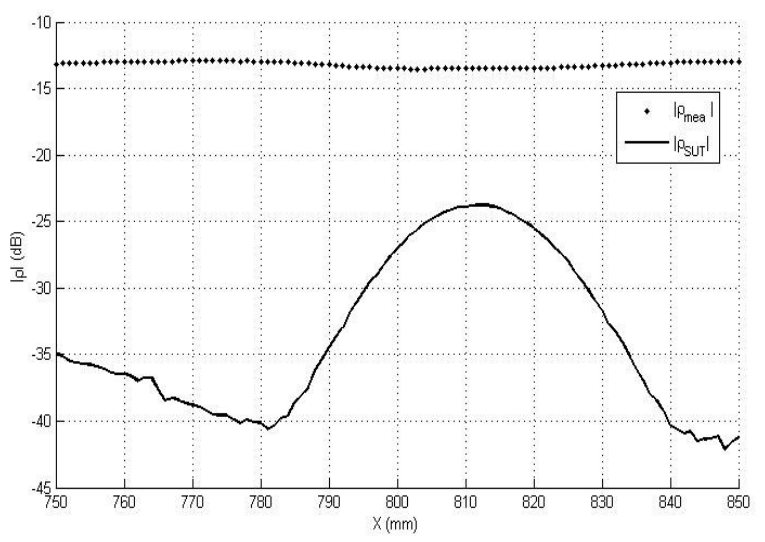

Fig. 8 Measurement of the SUT reflection coefficient of a plastic nut on a metal plate.

\section{CONCLUSION}

A FTA is a planar quasi-transparent lens that concentrates the energy of the feed at a point in the near-field of the lens, which improves the sensitivity of a detection system. This contribution proposes the use of a FTA to detect small objects on a reflective plate. In this situation, the objects cannot be detected using a typical system. However, the FTA increases the incident radiation on the target and makes possible its detection. This concept has been checked to detect a metal screw, a metal washer and a plastic nut on a metal plate. The FTA system measures an increase of the reflection coefficient variation of at least $5 \mathrm{~dB}$ when the object appears in the measurement scanning.

\section{ACKNOWLEDGMENT}

The present work has been developed under the support of the FPI pre-doctoral grant with ref no. BES-2012-053154, the project MIRIIEM with ref. TEC2014-540005-P of the Ministerio de Economía y Competitividad, and the research project of the Gobierno del Principado de Asturias / FEDER with ref. GRUPIN14-114.

\section{REFERENCES}

[1] C.G. Ryan, M.R. Chararmir, J. Shaker, J.R. Bray, Y.M.M. Antar, and A. Ittipiboon, "Wideband transmitarray using dual-resonant double square Rings", IEEE Trans. on Antenna Propag, vol. 58, no. 5, pp. 1486-1493, May 2010.

[2] W. Pan, C. Huang, X. Ma, B. Jiang, and X. Luo, "A dual linearly polarized transmitarray element with 1-Bit phase resolution in X-band", IEEE Antennas and Wireless Propag.Letters, vol. 14, pp. 167-170, 2015.

[3] A. Buffi, AA. Serra, P. Nepa, H.-T. Chou, and G. Manara, "A Focused Planar Microstrip Array for 2.4GHz RFID Readers", IEEE Trans Antenna Propag., vol. 58, no. 5, pp. 1536-1544, May 2010.

[4] K.D. Stephan, J.B. Mead, D.M. Pozar, L. Wang, and J.A. Pearce, “A Near Field Focused Microstrip Array for a Radiometric Temperature Sensor", IEEE Trans Antenna Propag., vol. 55, no. 4, pp. 1199-1203, April 2007.

[5] S. Karimkashi and A.A. Kishk, "Focused Microstrip Array Antenna using a Dolf-Chebysheff Near-Field Design", IEEE Trans Antenna Propag., vol. 57 , no. 12 , pp. 3813-3820, Dec. 2009. 
[6] M. Bogosanovic and A.G. Williamson, "Microstrip Antenna Array With a Beam Focused in the Near-Field Zone for Application in Noncontact Microwave Industrial Inspection", IEEE Trans Antenna Propag., vol. 56, no. 6, pp. 2186-2195, Dec. 2007.

[7] S. Karimkashi, and A.A. Kishk, "Focusing properties of Fresnel zone plates lens antennas in the Near-Field region", IEEE Trans Antenna Propag., vol. 59, no. 5, pp. 1481-1487, May 2011.

[8] E. G. Plaza, G. León, S. Loredo, and F. Las-Heras, "Near-field focusing transmitarray lens", 9th European Conference on Antennas and Propagation (EuCAP), Lisbon, Apr. 2015

[9] E. G. Plaza, G. León, S. Loredo, F. Las-Heras, A.Arboleya, C. M. Suárez, M. R. Pino "An ultrathin 2-bit Near Field Transmitarray lens", IEEE Antennas and Wireless Prop. Letters, Vol. 16. 2017. 